\title{
Quantization with operators appropriate to shapes of trajectories and classical perturbation theory
}

\author{
T. Uzer ${ }^{a)}$ and R. A. Marcus \\ Arthur Amos Noyes Laboratory of Chemical Physics, ${ }^{\text {b) }}$ California Institute of Technology, Pasadena, \\ California 91125
}

(Received 5 July 1984; accepted 18 July 1984)

\begin{abstract}
Quantization is discussed for molecular systems having a zeroth order pair of doubly degenerate normal modes. Algebraic quantization is employed using quantum operators appropriate to the shape of the classical trajectories or wave functions, together with Birkhoff-Gustavson perturbation theory and the Weyl correspondence for operators. The results are compared with a previous algebraic quantization made with operators not appropriate to the trajectory shape. Analogous results are given for a uniform semiclassical quantization based on Mathieu functions of fractional order. The relative sensitivities of these two methods (AQ and US) to the use of operators and coordinates related to and not related to the trajectory shape is discussed. The arguments are illustrated using principally a Hamiltonian for which many previous results are available.
\end{abstract}

\section{INTRODUCTION}

Many semiclassical quantization schemes have been designed for systems (or subsystems) of few coordinates in recent years, having or not having internal resonances. Resonances tend to distort the shape of the classical trajectories and the wave functions; and so create problems for some quantization methods, over and above those present for nonresonant systems. In the present article, several quantization schemes are considered for a system which has a 1:1 resonant Hamiltonian in its quadratic terms and where this degeneracy is removed by anharmonic couplings. The Hamiltonian and the classical operators are summarized in Sec. II, together with a classical Birkhoff-Gustavson perturbation expression. The desirability of using as quantum mechanical operators those which are particularly appropriate to the "shape" of the wave functions or of the corresponding classical trajectories is illustrated. An analogous conclusion was drawn in our semiclassical quantization, ${ }^{1,2}$ where the classical actions or quantization paths chosen were appropriate to the shape of the classical trajectories.

In Sec. III, the "algebraic" quantization method $(A Q),{ }^{3,4}$ which is based on the use of Birkhoff-Gustavson perturbation method ${ }^{5}$ (or on the classical "averaging" meth$\mathrm{od}^{6}$ ), is considered instead of the semiclassical method. Suitable Hamiltonian operators are introduced via the Weyl correspondence, ${ }^{4,7}$ based on quantum operators used by Louck and Shaffer ${ }^{8}$ in their quantum mechanical treatment of the doubly degenerate harmonic oscillator problem. These operators are appropriate to the shape of the classical "precessing" trajectories ${ }^{1}$ in this 1:1 resonant system. The results of this calculation are then contrasted with those obtained ${ }^{4}$ using instead "Cartesian operators". The former prove to yield the final results more straightforwardly, including various splittings of degeneracies. A related behavior has been noted previously in the spectroscopy literature. ${ }^{9}$

\footnotetext{
a) Present address: Department of Chemistry, University of Colorado, Boulder, Colorado 80309.

b) Contribution No. 7049.
}

The utility of the present AQ treatment is illustrated using the Henon-Heiles Hamiltonian, since many previous results are available for it for comparison. Section II contains a summary of the previous work on that system ${ }^{1,10,11}$ relevant to the present study.

For comparison with the AQ results, the uniform semiclassical (US) quantization ${ }^{12}$ of the same system is given in Sec. IV, using Birkhoff-Gustavson perturbation theory ${ }^{5}$ as before but introducing a quantization procedure which involves Mathieu functions ${ }^{13}$ of fractional order. The latter is performed with action variables 1,11 ("polar actions") appropriate to, and actions ${ }^{10}$ (Cartesian actions) not appropriate to the shape of the precessing trajectories. The superiority of the former is once again evident in the results. Indeed, in one previous semiclassical study where Cartesian actions were used, ${ }^{10}$ the quantization was effected using integration paths appropriate to the trajectory shapes.

The various results are discussed in Sec. V. The equivalence, not previously noted, between the Henon-Heiles Hamiltonian and Hamiltonians of molecular spectroscopic interest is also discussed in Sec. V. The anharmonicities in this potential split the overtones of the normal modes, but not the fundamentals. ${ }^{14}$ Results of the present paper are summarized in Sec. VI.

\section{MODEL SYSTEM}

The model system chosen for the present calculations is the Henon-Heiles Hamiltonian

$$
H=\left(p_{1}^{2}+q_{1}^{2}+p_{2}^{2}+q_{2}^{2}\right) / 2+\lambda q_{1}\left(q_{2}^{2}-q_{1}^{2} / 3\right) .
$$

Semiclassical quantization of this system was introduced by Noid and Marcus. ${ }^{1}$ They used as quantum numbers the principal quantum number $n$ (related to the total action) and the vibrational angular momentum quantum number $l$. They also observed that whereas some $(n, \pm l)$ pairs were degenerate, those having the same $n$ and $l= \pm 3 k$ should show a splitting when $k$ is an integer. Their "primitive" semiclassical calculation led to such $\pm 3 k$ pairs being apparently degenerate and they noted the need for a uniform connection 
formula to account for the missing splittings. Variational quantum mechanical results for the energy levels were given there and by Nordholm and Rice. ${ }^{15}$

The Birkhoff-Gustavson perturbation theory ${ }^{5}$ (BGPT), which consists of a series of canonical transformations that converts the classical Hamiltonian to a "normal" form, was first applied to the same system by Swimm and Delos. ${ }^{10}$ Their treatment gave most of the energy levels accurately. It was not uniform and so did not treat certain splittings. A uniform treatment using BGPT was subsequently given by Jaffe and Reinhardt. ${ }^{11}$ Both sets of authors used curvilinear properties analogous to those used in Ref. 1.

The (BGPT) normal form of the Hamiltonian (2.1) is expressed as ${ }^{5,10,11}$

$$
H=H_{0}+\lambda^{2} H_{2}+\lambda^{4} H_{4}+\lambda^{6} H_{6}+O(8),
$$

where $H_{0}$ and $H_{2}$ are

$$
\begin{aligned}
H_{0}= & \left(p_{1}^{2}+q_{1}^{2}+p_{2}^{2}+q_{2}^{2}\right) / 2, \\
H_{2}= & -\frac{5}{12}\left[\left(p_{1}^{2}+q_{1}^{2}\right) / 2\right]^{2}-\frac{5}{12}\left[\left(p_{2}^{2}+q_{2}^{2}\right) / 2\right]^{2} \\
& +\left(p_{1}^{2}+q_{1}^{2}\right)\left(p_{2}^{2}+q_{2}^{2}\right) / 12 \\
& -7\left[\left(p_{1} p_{2}+q_{1} q_{2}\right)^{2}-\left(p_{1} q_{2}-p_{2} q_{1}\right)^{2}\right] / 24 .
\end{aligned}
$$

One may express $H$ in terms of the four functions defined in Ref. 11:

$$
\begin{aligned}
& N_{0}=\left(p_{1}^{2}+q_{1}^{2}+p_{2}^{2}+q_{2}^{2}\right) / 2, \\
& N_{1}=\left(p_{1}^{2}+q_{1}^{2}\right) / 2-\left(p_{2}^{2}+q_{2}^{2}\right) / 2, \\
& N_{2}=p_{1} q_{2}-p_{2} q_{1}, \quad N_{3}=p_{1} p_{2}+q_{1} q_{2} .
\end{aligned}
$$

In the present article, $N_{0}$ and $N_{1}$ will be referred to as the "Cartesian" operators or actions and $N_{0}$ and $N_{2}$ as the "polar" operators or actions, $N_{2}$ being a vibrational angular momentum.

Equations (2.3) and (2.4) become

$$
\begin{aligned}
& H_{0}=N_{0}, \\
& H_{2}=-\left[3 N_{0}^{2}+7\left(N_{1}^{2}+N_{3}^{2}-N_{2}^{2}\right)\right] / 24 .
\end{aligned}
$$

$\mathrm{H}_{2}$ can be converted to a form which is diagonal in an $|n, l\rangle$ basis given later, if one introduces the classical relation

$$
N_{0}^{2}=N_{1}^{2}+N_{2}^{2}+N_{3}^{2} \text {. }
$$

$\mathrm{H}_{2}$ can then be rewritten as

$$
H_{2}=\left(7 N_{2}^{2}-5 N_{0}^{2}\right) / 12 \text {. }
$$

Similarly, the higher order terms are given as ${ }^{11}$

$$
\begin{aligned}
H_{4}= & -\left(67 N_{0}^{3}+21 N_{0} N_{2}^{2}\right) / 432 \\
& +7\left[4 N_{1}^{3}+3\left(N_{2}^{2}-N_{0}^{2}\right) N_{1}\right] / 18 \\
H_{6}= & 2093\left[4 N_{1}^{3}+3\left(N_{2}^{2}-N_{0}^{2}\right) N_{1}\right] N_{0} / 2160 \\
& -35 N_{0} N_{1} N_{2}^{2} / 648+35 N_{2}^{2}\left(2 N_{1}^{2}+N_{2}^{2}-N_{0}^{2}\right) / 1458 \\
& -\left(42229 N_{0}^{4}+458682 N_{0}^{2} N_{2}^{4}\right. \\
& \left.-575855 N_{2}^{4}\right) / 155520,
\end{aligned}
$$

when Eq. (2.8) is used. These classical equations for the normal form of the Hamiltonian are used in the following sections.

\section{ALGEBRAIC QUANTIZATION (AQ) OF THE NORMAL FORM}

The AQ method, which combines classical and quantum mechanical techniques to find the discrete spectrum of a Hamiltonian, was introduced by Sanders ${ }^{3}$ and Robnik. ${ }^{4}$ First, BGPT (or in Ref. 3 an equivalent "averaging" meth$\left.\mathrm{od}^{6}\right)$ was used to find the normal form of the classical Hamiltonian as above. ${ }^{4}$ The various perturbation terms, which are given in terms of coordinates and momenta, were then converted to quantum mechanical operators using ladder operators. The transition from commuting classical quantities to noncommuting operators was effected by the Weyl transformation ${ }^{7}$ which states, for instance, that the quantum mechanical operator corresponding to a classical expression is

$$
q^{m} p^{n} \leftrightarrow 2^{-n} \sum_{l=0}^{n} \frac{n !}{l !(n-l) !} P^{l} Q^{m} P^{n-1},
$$

where capital letters on the right denote operators. The quantization was then completed within the framework of second quantization. The correspondence in Eq. (3.1) is not unique. Questions concerning it are noted in Ref. 4.

The operator expressions that result from combining classical perturbation expressions, such as Eqs. (2.9) to (2.11), with formulas similar to Eq. (3.1) can be complicated and their evaluation tedious. Moreover, the diagonalization that is required by the second quantization involves progressively larger matrices as the principal quantum number $n$ increases, thereby adding to the effort involved. Fortunately, rather good results have been obtained for low lying states at even low orders, as was demonstrated by Sanders ${ }^{3}$ for the Fermi resonance and by Robnik ${ }^{4}$ for the Henon-Heiles system.

The aim in the present paper is not to give the most accurate solution of the present problem using AQ, by proceeding to high perturbations, for example, but rather to compare and contrast some features of the present AQ solution using polar operators with that ${ }^{3,4}$ given previously and based on Cartesian operators, and to compare both with various semiclassical ${ }^{1,10,11}$ and, hence, non-AQ quantizations. AQ quantization with polar operators is treated in Sec. III A below. For comparison, the Cartesian harmonic oscillator operators used by Sanders, ${ }^{3}$ Robnik, ${ }^{4}$ and in several calculations below are given in Sec. III B.

\section{A. Algebraic quantization of the normal form using polar operators' basis}

To express the various operators in the normal form in terms of the ladder operators in the present basis, we utilize operators $\xi$ and $\eta$, used by Louck and Shaffer ${ }^{8}$ for a twofold degenerate harmonic oscillator:

$$
\begin{aligned}
& \xi \equiv p_{+}+i q_{+}, \quad \xi^{\dagger} \equiv p_{-}-i q_{-}, \\
& \eta \equiv-p_{-}-i q_{-}, \quad \eta^{+} \equiv-p_{+}+i q_{+},
\end{aligned}
$$

where $\xi^{\dagger}$ and $\eta^{\dagger}$ are adjoints of $\xi$ and $\eta$ and where

$$
q_{ \pm} \equiv q_{1} \pm i q_{2}, \quad p_{ \pm}=p_{1} \pm i p_{2} \text {. }
$$

One choice of phase factors for the polar operators' basis, the eigenfunctions $|n, l\rangle$, is expressed by ${ }^{8}$ 


$$
\begin{aligned}
& \xi|n, l\rangle=-i[2(n+l+2)]^{1 / 2}|n+1, l+1\rangle, \\
& \eta|n, l\rangle=-i[2(n-l+2)]^{1 / 2}|n+1, l-1\rangle, \\
& \xi^{\dagger}|n, l\rangle=i[2(n-l)]^{1 / 2}|n-l, l-1\rangle, \\
& \eta^{\dagger}|n, l\rangle=i[2(n-l)]^{1 / 2}|n-1, l+1\rangle .
\end{aligned}
$$

The commutation relations of these operators are

$$
\begin{aligned}
& {\left[\xi^{\dagger}, \xi\right]=4, \quad\left[\xi^{\dagger}, \eta\right]=0, \quad\left[\xi^{\dagger} \xi, \eta^{\dagger} \eta\right]=0,} \\
& {\left[\eta^{\dagger}, \eta\right]=4, \quad[\xi, \eta]=0 .}
\end{aligned}
$$

In terms of these operators, the coordinate and momentum operators are given as

$p_{1}=\left(\xi-\eta+\xi^{\dagger}-\eta^{\dagger}\right) / 4, \quad q_{1}=i\left(\xi^{\dagger}-\eta^{\dagger}-\xi+\eta\right) / 4$,

$p_{2}=i\left(\xi^{\dagger}+\eta^{\dagger}-\xi-\eta\right) / 4, q_{2}=-\left(\xi+\eta+\xi^{\dagger}+\eta^{\dagger}\right) / 4$.

Using these relations the various operators $N_{i}$ in Eq. (2.5) can be found in terms of these ladder operators:

$$
\begin{aligned}
& N_{0}=\left(\xi^{\dagger} \xi+\eta^{\dagger} \eta-4\right) / 4, \quad N_{1}=-\left(\xi \eta^{\dagger}+\eta \xi^{\dagger}\right) / 4, \\
& N_{2}=\left(\eta^{\dagger} \eta-\xi^{\dagger} \xi\right) / 4, \quad N_{3}=i\left(\xi \eta^{\dagger}-\eta \xi^{\dagger}\right) / 4 .
\end{aligned}
$$

With Eq. (3.7) the $H_{i}$ in Eqs. (2.6) to (2.11) can also be expressed in terms of the ladder operators.

The effect of the operators $N_{i}$ on a basis set state $|n, l\rangle$ is given by

$$
\begin{aligned}
N_{0}|n, l\rangle= & (n+1)|n, l\rangle, \\
N_{1}|n, l\rangle= & -\frac{1}{2}[\sqrt{(n-l)(n+l+2)}|n, l+2\rangle \\
& +\sqrt{(n+l)(n-l+2)}|n, l-2\rangle], \\
N_{2}|n, l\rangle= & -l|n, l\rangle, \\
N_{3}|n, l\rangle=\frac{i}{2} & {[\sqrt{(n-l)(n+l+2)}|n, l+2\rangle} \\
& -\sqrt{(n+l)(n-l+2)}|n, l-2\rangle] .
\end{aligned}
$$

\begin{tabular}{|c|c|c|}
\hline & \multicolumn{2}{|c|}{ Splitting } \\
\hline & $(3, \pm 3)$ & $(6, \pm 6)$ \\
\hline $\begin{array}{l}\text { Exact }^{\mathrm{d}} \\
|n, l\rangle \text { basis, second order } \\
|n, l\rangle \text { basis, fourth order } \\
|n, l\rangle \text { basis, sixth order } \\
\left|n_{1}{ }^{\mathrm{a}}, n_{2}\right\rangle \text { basis, second order } \\
\left|n_{1}, n_{2}\right\rangle \text { basis, fourth order } \\
\text { US with vibrational } \\
\text { angular momentum } \\
\text { (Table IV) }\end{array}$ & $\begin{array}{c}0.0034 \\
0 \\
0.0029 \\
0.0033 \\
0 \\
0.0029 \\
0.0039\end{array}$ & $\begin{array}{c}0.0006 \\
0 \\
0.0003 \\
0.0005 \\
0 \\
0.0003 \\
0.0008\end{array}$ \\
\hline
\end{tabular}

One sees from Eq. (3.8) that in the $|n, l\rangle$ basis only $N_{0}$ and $N_{2}$ are diagonal. The effect of the $H_{i}$ is similarly obtained. For example, Eqs. (2.7) and (3.8) yield

$$
\begin{aligned}
H_{2}|n, l\rangle= & \left\{n+1+\left(\lambda^{2} / 24\right)\right. \\
& \left.\times\left[14 l^{2}-10(n+1)^{2}+7\right]\right\}|n, l\rangle,
\end{aligned}
$$

TABLE II. Splitting of $l= \pm 3 k$ levels of Henon-Heiles system using the AQ method.

${ }^{\text {a }}$ That is, $H_{0}$ and $H_{2}$ terms.

${ }^{\mathrm{b}} \mathrm{H}_{0}, \mathrm{H}_{2}$, and $\mathrm{H}_{4}$ terms.

${ }^{\mathrm{c}} \mathrm{H}_{0}, \mathrm{H}_{2}, \mathrm{H}_{4}$, and $H_{6}$ terms.

${ }^{\mathrm{d}}$ Reference 1 .

i.e., $H_{2}$ is diagonal in the $|n, l\rangle$ representation. Equation (3.9) is used to calculate the states for $n \leqslant 2$, and yields the $\mathrm{AQ}^{c}$ results given in Table I. Also given, to explain some aspects, are $A Q^{d}$ results obtained using the approximation for the quantum operators contained in Eq. (2.8). Thereby, Eqs. (2.9) and (3.8) are used, leading again to Eq. (3.9) but with the 7 missing. Exact and semiclassical results are also given in Table I for comparison.

\begin{tabular}{|c|c|c|c|c|c|c|}
\hline$(n, l)$ & $\mathrm{QM}^{\mathrm{a}}$ & $\begin{array}{c}\mathbf{A Q}^{\mathbf{b}} \\
\text { (Cartesian) }\end{array}$ & $A Q^{c}$ & $A Q^{d}$ & $\mathrm{SC}^{e}$ & $\mathbf{U S}^{f}$ \\
\hline$(0,0)$ & 0.9986 & 0.9986 & 0.9984 & 0.9948 & 0.9947 & 0.9966 \\
\hline$(1, \pm 1)$ & 1.9901 & 1.9901 & 1.9901 & 1.9863 & 1.9863 & 1.9868 \\
\hline$(2,0)$ & 2.9562 & 2.9562 & 2.9568 & 2.9532 & 2.9506 & 2.9558 \\
\hline$(2, \pm 2)$ & 2.9853 & 2.9853 & 2.9859 & 2.9823 & 2.9815 & 2.9812 \\
\hline
\end{tabular}

The splitting of the $l= \pm 3 k$ terms obtained with AQ and the $|n, l\rangle$ basis is treated in Appendix A and results are given in Table II. A leading term in determining the splitting using the $|n, l\rangle$ basis is identified in Appendix A, namely $N_{5}$, defined by

$$
N_{5} \equiv 4 N_{1}^{3}+3\left(N_{2}^{2}-N_{0}^{2}\right) N_{1} .
$$

It constitutes the off-diagonal part of $H_{4}$ in Eq. $(2.10)$ and also occurs in $H_{6}$.

\section{B. Quantization of the normal form using Carteslan operators' basis}

For this basis the usual harmonic oscillator raising and lowering operators are introduced:

TABLE I. Comparison of quantum, AQ [without and with Eq. (2.8)] and semiclassical results for the HenonHeiles system.

"Exact results from a large basis set quantum mechanical variational calculation in Ref. 1.

${ }^{b}$ Reference 4. Equation (3.17) is used, but not Eq. (2.8). These results differ slightly from those in Ref. 4, even though the equations there agree with ours. We believe some slight error occurred in the numerical calculations there.

${ }^{c}$ Equation (3.9) is used, but not Eq. (2.8).

Equations (2.8), (2.9), and (3.8) are used.

' Reference 1, classical trajectory data are used, with primitive semiclassical quantization. The latter quantization was crudest for the $l=0$ states.

${ }^{\mathrm{f}}$ Reference 11. These uniform semiclassical results were obtained using terms in $H$ up to and including $H_{6}$. 


$$
\begin{aligned}
& z_{j}^{\dagger}\left|n_{j}\right\rangle=\sqrt{n_{j}+1}\left|n_{j}+1\right\rangle, \\
& z_{j}\left|n_{j}\right\rangle=\sqrt{n_{j}}\left|n_{j}-1\right\rangle .
\end{aligned}
$$

They are related to the original coordinate and momentum operators by

$$
q_{j}=\left(z_{j}+z_{j}^{\dagger}\right) / \sqrt{2}, \quad p_{j}=i\left(z_{j}^{\dagger}-z_{j}\right) / \sqrt{2} .
$$

In this basis, one finds the operators

$$
N_{0}=z_{1}^{\dagger} z_{1}+z_{2}^{\dagger} z_{2}+1
$$

and

$$
N_{1}=z_{1}^{\dagger} z_{1}-z_{2}^{\dagger} z_{2}
$$

to be diagonal, whereas

$$
N_{2}=i\left(z_{1} z_{2}^{\dagger}-z_{1}^{\dagger} z_{2}\right)
$$

and

$$
N_{3}=z_{1}^{\dagger} z_{2}+z_{1} z_{2}^{\dagger}
$$

are nondiagonal.

The normal form through second order is, from Eq. (3.5),

$$
\begin{aligned}
H_{0}+ & \lambda^{2} H_{2} \\
= & z_{1}^{\dagger} z_{1}+z_{2}^{\dagger} z_{2}+1-\lambda^{2}\left\{5\left[\left(z_{1}^{\dagger} z_{1}+\frac{1}{2}\right)^{2}+\left(z_{2}^{\dagger} z_{2}+\frac{1}{2}\right)^{2}\right]\right. \\
& \left.\quad-2\left(z_{1}^{\dagger} z_{1}+\frac{1}{2}\right)\left(z_{2}^{\dagger} z_{2}+\frac{1}{2}\right)+7\left[\left(z_{1} z_{2}^{\dagger}\right)^{2}+\left(z_{1}^{\dagger} z_{2}\right)^{2}\right]\right\} / 12
\end{aligned}
$$

The $z_{i} z_{j}^{\dagger}(i \neq j)$ terms in Eq. (3.17) couple states of the form $\left|n_{1}, n_{2}\right\rangle$ with $\left|n_{1} \pm 2, n_{2} \mp 2\right\rangle$. One sees that while $H_{2}$ is diag. onal in the $|n, l\rangle$ basis for the polar actions [i.e., as in Eq. (3.9)], it is not diagonal for the Cartesian actions (i.e., for the $\left|n_{1}, n_{2}\right\rangle$ basis $)$.

Results ${ }^{4}$ are given in Table I for the eigenvalues of the Cartesian-based (3.17) for $n \leqslant 2$. Several results for the $(3, \pm 3)$ and $(6, \pm 6)$ states using this $\left|n_{1}, n_{2}\right\rangle$ basis are given in Table II. To illustrate further the difference between the use of the Cartesian and polar operators' basis sets (see Sec. III A), we consider in Table III the states having $n=3$, states not examined in Ref. 4: A $4 \times 4$ matrix for $\mathrm{H}_{2}$ was constructed, and was found to consist of two block-diagonal matrices, indicating that these states split into two sets of doubly degenerate states. The corresponding eigenvalues are given in Table III.

\section{UNIFORM SEMICLASSICAL QUANTIZATION (US) OF THE NORMAL FORM}

In the semiclassical treatment of Eq. (2.2) it is well known that the total action, $N_{0}$ in Eq. (2.5), is a constant of

TABLE III. Cartesian AQ eigenvalues of Henon-Heiles system compared with exact eigenvalues for $n=3$.

\begin{tabular}{lcc}
\hline$E$ & $\mathrm{QM}^{\mathrm{a}}$ & $\begin{array}{c}\text { Eigenvalue } \\
\text { from } \mathrm{AQ}^{\mathrm{b}}\end{array}$ \\
\hline$E_{3,+1}$ & 3.9260 & 3.9276 \\
$E_{3, \pm 1}$ & 3.9824 & 3.9859 \\
$\frac{1}{2}\left(E_{3,+3}+E_{3,-3}\right)-E_{3, \pm 1}$ & 3.9858 & 0.0583 \\
\hline
\end{tabular}

${ }^{a}$ Exact results (Ref. 1). ${ }^{\mathrm{b}}$ Using $\mathrm{H}_{2}$ and a Cartesian basis. the motion. The second action variable can either be chosen as $N_{1}$, as in Ref. 10, or $N_{2}$, as in Ref. 11. In both cases, Eq. (2.1) provides a functional relationship

$$
E=f\left(\lambda, N_{0} ; I_{\alpha}, \alpha\right)
$$

between the second action $N_{1}$ or $N_{2}$, written now as $I_{\alpha}$, and its conjugate angle $\alpha$. This relation can then be inverted (numerically when a perturbation theory of higher order is used) and used to quantize the integral $\oint I_{\alpha} d \alpha$ and to yield the energy levels $E$.

In comparing the choices for the second (variable) action, it should be noted that for the set $\left(N_{0}, N_{1}\right)$, the time variation of the action $N_{1}$ is (by Hamilton's equations) proportional to $\lambda^{2}$ [cf. Eqs. (B3) and (B4)], whereas the same variation for $N_{2}$ is proportional to $\lambda^{4}$ [cf. Eq. (4.5) below]. Consequently, $N_{2}$ is a "better" action variable than $N_{1} . N_{0}$ is a good action variable for both choices.

In formulating a uniform semiclassical treatment (US) the ideas we employed in a uniform semiclassical theory of avoided crossings ${ }^{12}$ are utilized. There, the resulting model Hamiltonian in action-angle variables contained a cosine barrier and was quantized using Mathieu functions ${ }^{13}$ of fractional order. (Alternatively, phase integral quantization has also been used to obtain a US approximation for cosine barrier problems, ${ }^{16,17}$ but for a simple cosine potential the present method is as simple and can be more accurate, as in Ref. 17 and in Table IV later.) In the following sections, the method of Ref. 12 is used to quantize the part of Eq. (2.2) obtained by truncating that series as soon as a trigonometric term in the "slow variable" arises in the perturbation series. A procedure identical to that used in Ref. 12 again results in Mathieu functions of fractional order. The quantization was performed with the two sets of actions discussed above, for purposes of comparison. The quantization with the polar actions $\left(N_{0}, N_{2}\right)$ is described first, the quantization using the Cartesian actions $\left(N_{0}, N_{1}\right)$ being given in Appendix B.

When quantizing with $N_{0}, N_{2}$ and the angle $\varphi$ conjugate to $N_{2}$, the variables $N_{i}$ can be expressed as

$$
\begin{aligned}
& N_{0} \equiv I, \quad N_{1}=\left(I^{2}-I_{\varphi}^{2}\right)^{1 / 2} \cos 2 \varphi, \\
& N_{2} \equiv I_{\varphi}, \quad N_{3}=\left(I^{2}-I_{\varphi}^{2}\right)^{1 / 2} \sin 2 \varphi .
\end{aligned}
$$

When these transformations are introduced into Eqs. (2.9) to (2.11), $H_{4}$ is the first term that contains a trigonometric term in $\varphi$. If $H$ is truncated after terms that are proportional to $\lambda^{4}$, the resulting approximate Hamiltonian is

$$
H=H_{A}(I)+H_{B}\left(I_{\varphi}, \varphi, I\right),
$$

where

$$
H_{A}(I)=I-5 \lambda^{2} I^{2} / 12-67 \lambda^{4} I^{3} / 432
$$

and

$$
\begin{aligned}
H_{B}\left(I_{\varphi}, \varphi, I\right)= & 7 \lambda^{2} I_{\varphi}^{2} / 12-7 \lambda^{4} I_{\varphi}^{2} I / 144 \\
& +\frac{7}{18} \lambda^{4}\left(I^{2}-I_{\varphi}^{2}\right)^{3 / 2} \cos 6 \varphi .
\end{aligned}
$$

$I$ is exactly and $I_{\varphi}$ is approximately a constant of the motion. The $\left(I^{2}-I_{\varphi}^{2}\right)^{3 / 2}$ factor in Eq. (4.5) can, in one approximation, be replaced by a suitable average value (denoted by \langle\rangle ). To obtain later the Mathieu equation in a standard form, we then define 


$$
q=\lambda^{2}\left\langle\left(I^{2}-I_{\varphi}^{2}\right)^{3 / 2}\right\rangle / 27\left(1-\lambda^{2} I / 12\right)
$$

and replace $H$ by $E$. One then obtains

$$
\begin{aligned}
E= & H_{A}(I)+7 \lambda^{2}\left(1-\lambda^{2} I / 12\right) I_{\varphi}^{2} \\
& +\frac{21}{2} \lambda^{2} q\left(1-\lambda^{2} I / 12\right) \cos 6 \varphi .
\end{aligned}
$$

Upon rearranging, $I_{\psi}$ is expressed as a function of $\varphi$ :

$$
\begin{aligned}
I_{\Phi}^{2}+ & {\left[H_{A}(I)-E\right]\left[7 \lambda^{2}\left(1-\lambda^{2} I / 12\right) / 12\right]^{-1} } \\
& +18 q \cos 6 \varphi=0 .
\end{aligned}
$$

To introduce a uniform semiclassical quantization by converting Eq. (4.8) to a differential equation, one replaces the actions by differential operators. ${ }^{12}$ The resulting equation is one-dimensional because $I$ is now a constant of the motion and can be replaced by its constant value. The replacement

$$
I_{\varphi} \rightarrow-i d / d \varphi
$$

gives the Schrödinger equation for the $\varphi$ wave function $F(\varphi)$ :

$$
d^{2} F(\varphi) / d \varphi^{2}+(9 a-18 q \cos 6 \varphi) F(\varphi)=0
$$

with

$$
a=4\left[E-H_{A}(I)\right]\left[21 \lambda^{2}\left(1-\lambda^{2} I / 12\right)\right]^{-1} .
$$

A change of variables:

$$
\begin{aligned}
& \alpha=3 \varphi, \quad \psi(\alpha) \equiv F(\varphi), \\
& I_{\alpha}=I_{\varphi} / 3, \quad d / d \alpha=(1 / 3) d / d \varphi
\end{aligned}
$$

transforms Eq. (4.10) into a standard Mathieu equation ${ }^{13}$

$$
d^{2} \psi(\alpha) / d \alpha^{2}+(a-2 q \cos 2 \alpha) \psi(\alpha)=0
$$

with the boundary condition ${ }^{18}$

$$
\psi(\alpha+\pi)=e^{i l \pi / 3} \psi(\alpha) .
$$

Here, $l$ is an integer and, when $q=0$, equals $I_{\varphi}^{(0)}$, the unperturbed vibrational angular momentum. ${ }^{18} I_{\psi}^{(0)}$ and hence $I_{\alpha}^{(0)}$ is an approximate constant of the motion, which would be constant if $q$ in Eq. (4.13) vanished.

The general solution of Eqs. (4.13) and (4.14) can be written in the Floquet form ${ }^{19}$

$$
\psi_{v}(\alpha)=e^{i v \alpha} P(\alpha),
$$

with $P(\alpha)$ being periodic in $\alpha$ with period $\pi$. From Eq. (4.15) one has

$$
\psi_{v}(\alpha+\pi)=e^{i v \pi} \psi_{v}(\alpha),
$$

comparison with Eq. (4.14) shows that the Floquet exponent $v$ (the order of the Mathieu function) is given by

$$
v=l / 3 \text {. }
$$

\begin{tabular}{|c|c|c|c|c|}
\hline $\begin{array}{l}\text { Quantum } \\
\text { numbers }\end{array}$ & $\begin{array}{l}\text { Symmetry } \\
\text { quantum } \\
\text { state }\end{array}$ & $\mathrm{QM}^{\mathrm{a}}$ & $\begin{array}{l}\mathrm{US}^{\mathrm{b}} \\
\Delta E\end{array}$ & $\begin{array}{l}\mathrm{US}^{\mathrm{c}} \\
\Delta E\end{array}$ \\
\hline$n \quad l$ & & & & \\
\hline $\begin{array}{ll}0 & 0\end{array}$ & $A$ & 0.9986 & 0.9948 & 0.9966 \\
\hline $1 \pm 1$ & $E$ & 1.9901 & 1.9863 & 1.9868 \\
\hline $2-0$ & $A$ & 2.9562 & 2.9525 & 2.9558 \\
\hline $2 \pm 2$ & $E$ & 2.9853 & 2.9816 & 2.9812 \\
\hline \multirow[t]{2}{*}{$3 \pm 1$} & $E$ & 3.9260 & 3.9233 & 3.9241 \\
\hline & & 3.9824 & 3.9785 & 3.9772 \\
\hline $3 \pm 3$ & $A$ & 3.9858 & 3.9824 & 3.9827 \\
\hline $\begin{array}{ll}4 & 0\end{array}$ & $A$ & 4.8702 & 4.8667 & 4.8731 \\
\hline $4 \pm 2$ & $E$ & 4.8987 & 4.8956 & 4.8942 \\
\hline $4 \pm 4$ & $E$ & 4.9863 & 4.9830 & 4.9821 \\
\hline \multirow[t]{2}{*}{$5 \pm 1$} & $E$ & 5.8170 & 5.8142 & 5.8202 \\
\hline & & 5.8670 & 5.8659 & 5.8608 \\
\hline $5 \pm 3$ & A & 5.8815 & 5.8790 & 5.8778 \\
\hline $5 \pm 5$ & $E$ & 5.9913 & 5.9893 & 5.9871 \\
\hline 60 & $A$ & 6.7379 & 6.7357 & 6.7440 \\
\hline $6 \pm 2$ & $E$ & 6.7649 & 6.7639 & 6.7636 \\
\hline \multirow[t]{2}{*}{$6 \pm 4$} & $E$ & 6.8354 & 6.8533 & 6.8493 \\
\hline & & 6.9989 & 6.9968 & 6.9954 \\
\hline $6 \pm 6$ & $\boldsymbol{A}$ & 6.9994 & 6.9977 & 6.9954 \\
\hline \multirow[t]{2}{*}{$7 \pm 1$} & $E$ & 7.6595 & 7.6584 & 7.6674 \\
\hline & & 7.6977 & 7.7034 & 7.6953 \\
\hline $7 \pm 3$ & $A$ & 7.7369 & 7.7345 & 7.7329 \\
\hline $7 \pm 5$ & $E$ & 7.8327 & 7.8361 & 7.8293 \\
\hline $7 \pm 7$ & $E$ & 8.0094 & 8.0090 & 8.0059 \\
\hline 80 & $A$ & 8.5541 & 8.5568 & 8.5638 \\
\hline $8 \pm 2$ & $E$ & 8.5764 & 8.5830 & 8.5881 \\
\hline \multirow{2}{*}{$8 \pm 4$} & $E$ & 8.6779 & 8.6806 & 8.6730 \\
\hline & & 8.8113 & 8.8199 & 8.8088 \\
\hline $8 \pm 6$ & $A$ & 8.8152 & 8.8236 & 8.8132 \\
\hline $8 \pm 8$ & $E$ & 9.0217 & 9.0234 & 9.0193 \\
\hline
\end{tabular}

This order is fractional in general. The eigenvalues of the Mathieu equation corresponding to orders $\pm|v|$ are different when $v$ is an integer and are degenerate for fractional $v{ }^{13}$ This result shows directly that states with $l= \pm 3 k$ (with $k$ an integer) are split, whereas others remain degenerate.

If the eigenvalue $a$ in Eq. (4.13) corresponding to order $v$ is denoted by $a_{v}$, then the quantized energy levels are obtained from

$$
E_{v}=H_{A}(I)+21 \lambda^{2}\left(1-\lambda^{2} I / 12\right) a_{v} / 4,
$$

where Eq. (4.11) has been used. The eigenvalues $a_{v}$ are readily calculated. ${ }^{20}$
TABLE IV. The low-lying bound states of the Henon-Heiles system obtained from various calculations.

Exact results (Ref. 1).

${ }^{\mathrm{b}}$ Uniform semiclassical calculation using terms up and including $\lambda{ }^{4}$ and the approximation embodied in Eqs.(4.18) and (4.19).

'Uniform semiclassical calculation (Ref. 11) using terms up to and including $\lambda^{6}$.

The simplest approximation to $q$ in Eq. (4.6) is to replace the $I_{q}$ on the average by zero, thus obtaining

$$
q=\lambda^{2} I^{3} / 27\left(1-\lambda^{2} I / 12\right) .
$$

Other approximations can be devised. The energy levels obtained using the $q$ from Eq. (4.19) are given in Table IV. In Table $\mathrm{V}$, the splittings of the $l= \pm 3 k$ states, calculated from Eqs. (4.18) and (4.19), are compared with the exact results in Ref. 1. They are also compared with the results of Ref. 11, which employed a higher order perturbation theory for the Hamiltonian plus phase integral (rather than Mathieu function) uniform semiclassical quantization.

For comparison with these results an analogous formulation based on Cartesian actions $\left(N_{0}, N_{1}\right)$ instead of the angular momentum ones $\left(N_{0}, N_{2}\right)$ is given in Appendix B. Calculations up to and including order $\mathrm{H}_{2}$ are reported in Table VI. The latter contains under US, the results of solving a Mathieu equation of fractional order, Eq. (B7) in Appendix B, and under SD the results of diagonalizing the same semiclassically based equation in a subspace of the $n=2$ states. Also shown in Table VI are results for $n=3$, treated similarly. 
TABLE V. Splittings of the $l= \pm 3 k$ levels of Henon-Heiles system, $k$ an integer.

\begin{tabular}{rllll}
\hline \hline $\begin{array}{c}\text { Quantum } \\
\text { numbers } \\
n \pm l\end{array}$ & $\begin{array}{c}\mathrm{QM}^{\mathrm{a}} \\
\Delta E\end{array}$ & $\begin{array}{c}\mathrm{AQ}^{\mathrm{b}} \\
\Delta E\end{array}$ & $\begin{array}{c}\mathrm{US}^{\mathrm{c}} \\
\Delta E\end{array}$ & $\begin{array}{c}\mathrm{US}^{\mathrm{d}} \\
\Delta E\end{array}$ \\
\hline $3 \pm 3$ & 0.0034 & 0.0033 & 0.0039 & 0.0055 \\
$5 \pm 3$ & 0.0145 & 0.0138 & 0.0131 & 0.0170 \\
$7 \pm 3$ & 0.0392 & 0.0364 & 0.0311 & 0.0376 \\
$9 \pm 3$ & 0.085 & 0.0765 & 0.0605 & 0.0638 \\
$11 \pm 3$ & 0.165 & 0.140 & 0.104 & 0.139 \\
$13 \pm 3$ & 0.294 & 0.235 & 0.161 & 0.234 \\
& & & & \\
$6 \pm 6$ & 0.0006 & & 0.0008 & 0.0005 \\
$8 \pm 6$ & 0.0039 & & 0.0038 & 0.0044 \\
$10 \pm 6$ & 0.017 & & 0.0121 & 0.0190 \\
$12 \pm 6$ & 0.157 & & 0.0294 & 0.158 \\
& & & & \\
$9 \pm 9$ & 0.001 & & 0.0002 & 0.0001 \\
$11 \pm 9$ & 0.002 & & 0.0042 & 0.0002 \\
$13 \pm 9$ & $\ldots$ & & 0.0057 & 0.0077 \\
& & & & \\
$12 \pm 12$ & 0.01 & & 0.0001 & 0.0001 \\
\hline \hline
\end{tabular}

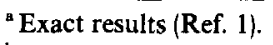

'Matrix $2 \times 2$ diagonalization with polar operators' basis, using terms up to and including $\lambda^{4}$ [Eqs. (A4) and (A5)] for $l= \pm 3$.

${ }^{c}$ Present results using terms up to and including $\lambda^{4}$ [Eqs. (4.18) and (4.19)].

${ }^{d}$ Uniform semiclassical calculation of Ref. 11 , using terms up to and including $\lambda^{6}$.

The Fermi resonance system ${ }^{2}$ provides another example of a use of the AQ method and is useful for a comparison with the uniform semiclassical method. The uniform semiclassical treatment given in Appendix $C$ is based on Cartesian actions. It yields the results listed under US in Table VII. They are compared there with the exact results, with AQ results, and with the "primitive" semiclassical results, the latter obtained from phase integrals calculated over appropriate curvilinear paths using classical trajectory data. ${ }^{2}$ In the $\mathrm{SC}^{\mathrm{d}}$ column in Table VII we have used a $2 \times 2$ matrix diagonalization of the same Hamiltonian as that used for the US results, namely the differential equation $(\mathrm{Cl} 16)$. In this diagonalization the two states $\left|n_{1}, n_{2}\right\rangle=|1,1\rangle$ and $|0,3\rangle$ served as a basis set, the (unnormalized) states being

TABLE VI. Comparison of various quantization schemes for the $H_{2}$ Hamiltonian for the Henon-Heiles system.

\begin{tabular}{cccc}
\hline \hline $\begin{array}{c}\text { Quantum } N \\
\text { numbers } \\
(n, 1)\end{array}$ & $\mathrm{QM}^{\mathrm{a}}$ & $\mathrm{US}^{\mathrm{b}}$ & $\mathrm{SD}^{\mathrm{c}}$ \\
\hline$(2,0)$ & 2.9562 & 2.9576 & 2.9550 \\
$(2, \pm 2)$ & 2.9853 & $\left\{\begin{array}{l}2.9898 \\
2.9921\end{array}\right.$ & 2.9859 \\
& & 3.9337 & 2.9878 \\
$(3, \pm 1)$ & 3.9260 & 3.9939 & 3.9258 \\
$(3, \pm 3)$ & $(3.9858$ & & \\
& 3.9824 & & \\
\hline
\end{tabular}

${ }^{a}$ Exact results (Ref. 1).

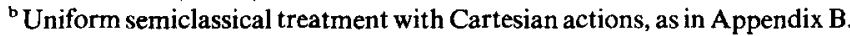

'Matrix diagonalization of Cartesian actions' case, semiclassical equation (B7), as in Appendix B.
TABLE VII. Fermi resonance system for $n=3$ states."

\begin{tabular}{cccccc}
\hline \hline State $(n, l)$ & $\mathrm{QM}^{\mathrm{b}}$ & $\mathrm{AQ}^{\mathrm{c}}$ & $\mathrm{SD}^{\mathrm{d}}$ & $\mathrm{US}^{\mathrm{e}}$ & $\mathrm{SC}^{\mathrm{f}}$ \\
\hline$(3 .+1)$ & 3.100 & 3.101 & 3.099 & 3.140 & 3.099 \\
$(3,-1)$ & 3.184 & 3.184 & 3.185 & 3.177 & 3.185 \\
\hline \hline
\end{tabular}

${ }^{2}$ Hamiltonian in Eq. (C1), with $\lambda=-\eta=-0.04$ and $\omega=1.4 ; n$ is $2 n_{x}$ $+n_{p} ; l$ is defined in Ref. 2.

${ }^{\text {"Ex }}$ Exact results in Table I of Ref. 2.

"Algebraic quantization (Ref. 3) for the Hamiltonian in Eq. (C1), as in Appendix C, calculated by D. Wardlaw (private communication).

" $2 \times 2$ matrix diagonalization of Cartesian actions' semiclassical equation (C16).

"Uniform semiclassical result using Eq. (C18).

${ }^{\text {f }}$ Primitive semiclassical result in Table I of Ref. 2 , using classical trajectories.

$|1,1\rangle=\exp (3 i \alpha)$ and $|0,3\rangle=\exp (i \alpha)$. Calculation of the matrix elements of $2 q \cos 2 \alpha$ in Eq. (C16) [e.g., as in Eq. (B13)] yields upon diagonalization the $\mathrm{SD}^{\mathrm{d}}$ values in Table VII.

\section{DISCUSSION}

\section{A. $A Q$ results}

The AQ results for $n \leqslant 2$ in Table I, obtained with the $|n, l\rangle$ basis and Eq. (3.9) for $\mathrm{H}_{2}$, agree with those calculated ${ }^{4}$ using Eq. (3.17) and a Cartesian basis $\left|n_{1}, n_{2}\right\rangle$. Both agree quite well with the exact results ${ }^{1}(\mathrm{QM})$ and, as one sees from Table I, they agree better with QM results than do earlier semiclassical ones ${ }^{1.10,11}$ calculated to higher order. This difference occurs even though the perturbation form of $\mathrm{H}_{2}$ and the corresponding operator are obtained from the same normal form as that used for the semiclassical result. The error appears to lie in the use of these semiclassical theories at low energies: In particular the effect of introducing into $A Q$ the classical identity (2.8) was examined. This identity is not exact for quantum operators. From the AQ results obtained using and not using Eq. (2.8) in Table I, it is seen that when Eq. (2.8) is used the AQ results are no longer an improvement over the semiclassical ones (cf. column $\mathrm{AQ}^{\mathrm{d}}$ in Table I). The off-diagonal elements of both sides of Eq. (2.8) are equal but the diagonal elements differ. ${ }^{21}$

\section{B. AQ splittings for $I= \pm 3 k$ states}

The splittings for $l= \pm 3 k$ states are given in Table II. One feature not obtained in a primitive semiclassical' trajectory calculation, but obtained in a uniform semiclassical calculation, " were these splittings. To see how the AQ method treats them, the simplest example, namely the $2 \times 2$ diagonalization of the Hamiltonian in the subspace of the $|n, l\rangle=|n, \pm l\rangle$ states, was considered:

The operator $H_{2}$ in Eq. (3.9) is not able to resolve the $l= \pm 3 k$ degeneracy because this $H_{2}$ is diagonal in the $|n, l\rangle$ basis. The use ${ }^{4}$ of Cartesian operators and terms in $H$ only up to $H_{2}$, which served so well in Ref. 4 for $n \leqslant 2$ (see also the present Table I) also does not yield the splittings of the $|n, l\rangle=|3, \pm 3\rangle$ states (Table II). It does yield, however, an excellent value for the energy difference between the mean of the eigenvalues for the $|3, \pm 3\rangle$ and $|3, \pm 1\rangle$ states (Table III). 
When the AQ method is used and $H_{4}$ terms are included in the matrix diagonalization a reasonable value is then obtained for the $l= \pm 3$ splitting, using the $|n, l\rangle$ or the $\left|n_{1}, n_{2}\right\rangle$ basis (Table II). The latter agree exactly with each other. However, the calculation using $H_{4}$ in the $\left|n_{1}, n_{2}\right\rangle$ basis is lengthy, because none of the operators in $\mathrm{H}_{4}$ can be identified with an operator that brings about the splitting. The splitting in the case of the $\left|n_{1}, n_{2}\right\rangle$ basis arises because the two $2 \times 2$ block matrices from before are slightly different due to $\mathrm{H}_{4}$, and one has to calculate all four eigenvalues to find the splitting. In Sec. III A where AQ was performed using the $|n, l\rangle$ basis the same result was obtained at this order, but the operators that lead to this splitting were identifiable. Relatively little effort (cf. Appendix A), was needed to continue to the next order and we found that the remaining discrepancy can almost totally be removed by $H_{6}$, as in Table II. The same procedure in the $\left|n_{1}, n_{2}\right\rangle$ basis would be a very lengthy task, indeed. For example, the calculation of the $l= \pm 6$ splittings in the $n=6$ subspace requires, in the Cartesian basis, the complete (through $H_{6}$ ) diagonalization of a $7 \times 7$ matrix, all elements of which must be calculated accurately. The advantages of a basis that conforms to the approximately "good" quantum numbers is once again evident. In summary, the $|n, l\rangle$ basis is computationally much more convenient than the $\left|n_{1}, n_{2}\right\rangle$ one. Interestingly enough, the $2 \times 2$ diagonalization for $\mathrm{AQ}$ leads (Table $\mathrm{V}$ ) to splittings for the $(n, \pm 3)$ states which are as good as or better than those obtained by a uniform semiclassical method in Ref. 11 which involves higher order (cf. below).

\section{Uniform semiclassical results with polar actions' basis}

The uniform semiclassical results (US) with the $|n, l\rangle$ basis are given in Tables IV and V. One sees that for the levels in Table IV (i.e., $n \leqslant 8$ ), with an energy up to more than half the dissociation energy, the uniform semiclassical results based on Eqs. (4.18) and (4.19) are quite accurate, both for the levels and the splittings, considering the approximations made to obtain the Mathieu equation. Indeed, the results are on the average comparable (somewhat more accurate in $\mathrm{Ta}$ ble IV and somewhat less accurate in Table V) than those in Ref. 11 , which employed terms up to and including $H_{6}$ instead of $\mathrm{H}_{4}$ and which solved a higher order polynomial for $I_{\varphi}$. The results calculated from Eqs. (4.18) and (4.19) in Table $V$ for the splitting of the $l= \pm 3 k$ levels also compare well with the exact results (QM), and with the results obtained in Ref. 11, which used the higher order Hamiltonian. The agreement obtained with $H_{4}$ and Eqs. (4.18) and (4.19) becomes somewhat worse in each sequence as $n$ increases, as in Table V. Presumably the higher order terms are needed at higher $n$ 's.

\section{Semiclassical results with Cartesian actions' basis and US or a curvilinear path}

The uniform semiclassical results in Table VI for $n=3$ show that the semiclassical results obtained with $\mathrm{H}_{2}$ and Cartesian actions, either using a uniform treatment or the diagonalization of $\mathrm{H}_{2}$ discussed at the end of Appendix B, do not agree well with the exact values (QM). Both lead to qual- itatively and quantitatively incorrect results. As was mentioned earlier, Swimm and Delos ${ }^{10}$ quantized the normal form of Hamiltonian (2.1) including $\mathrm{H}_{8}$ terms using Cartesian actions. Their results are appreciably better than the Cartesian uniform values given in Table VI. Part of the improvement may be due to the use of a high order perturbation theory, and as a result, avoiding the use of averaged actions in the couplings at low order. However, the main reason is to be sought in the special integration paths they employed to quantize their actions described below. As a test of this idea we used the Cartesian actions of Ref. 10 along a Cartesian instead of an appropriate curvilinear integration path. The results (not given here) were in poorer agreement with the exact quantum results and, indeed, were close to those obtained in Table VI with Cartesian actions plus uniform semiclassical theory.

We recall briefly the nature of the curvilinear paths used in Refs. 1 and 10 to make the semiclassical quantization: The normal form in Ref. 10 provided the authors with an implicit relation between $J, J^{\prime}$ (corresponding to the present $N_{0}$ and $N_{1}$ ) and conjugate angles $w$ and $w^{\prime}$, in the form

$$
E=\mathbf{f}\left(\lambda, J ; J^{\prime}, w^{\prime}\right),
$$

namely, their Eqs. (33a)-(33d). They quantized $J$ and $J^{\prime}$ through the relations

$\oint_{C} J d w=\oint_{c} \sum_{k} p_{k} d q_{k}, \quad \oint_{c^{\prime}} J^{\prime} d w^{\prime}=\oint_{c^{\prime}} \sum_{k} p_{k} d q_{k}$,

where for path $C$ one lets $w$ increase by one unit, keeping $w^{\prime}$ fixed, and for path $C^{\prime} w^{\prime}$ increases by one unit, keeping $w$ fixed. They then transformed the paths $C$ and $C^{\prime}$ to Cartesian phase space using equations similar to the present Eq. (B1). They were then able to deform these paths such that the path integrals over them were expressible in terms of the quantization paths of Noid and Marcus, ' namely Eqs. (5.3)-(5.5) for precessing trajectories

$$
\begin{aligned}
& \oint_{C} J d w=2 \pi(n+1), \\
& \oint_{C} J^{\prime} d w^{\prime}=2 \pi\left(n_{r}+1 / 2\right)=\pi(n-l+1),
\end{aligned}
$$

$n$ being the principal quantum number, and Eq. (5.5) instead of Eq. (5.4) for librating trajectories ${ }^{1,10}$

$$
\oint_{C^{\prime}} J^{\prime} d w^{\prime}=2 \pi l / 3 .
$$

These equations immediately related the quantization integrals (5.2) to the good quantum numbers $n, l$ of the system.

One sees that although quantization is best performed in coordinates appropriate to the shape of the trajectories, the disadvantages of using other schemes can be offset by using path integrals which are expressible in terms of the good quantum numbers of the system. This latter point is noteworthy, since it is usually difficult to find a simple system of (nonnumerically determined) coordinates and operators that reflect trajectory shapes, except in special cases.

\section{E. Uniform and AQ results for Fermi resonance system}

Uniform semiclassical results for the Fermi resonance system, given in Table VII and obtained with Cartesian ac- 
tions in Appendix $\mathrm{C}$, are in poorer agreement with the exact results $(Q M)$ than are the results ${ }^{3}$ obtained there with the $A Q$ method. A reason for this behavior is evident from the analysis in Appendix C: The perturbation expression obtained in terms of the Cartesian actions now has a singularity in the "barrier height" $q$ at $\lambda=0$, as in Eqs. (C12) and (C13), where $\lambda$ is a perturbation parameter. [If one introduced instead of $I_{\alpha}$ and $\alpha$ in Eq. (C9) $I_{\gamma}=\lambda I_{\alpha}$ and $\gamma=\alpha / \lambda$ to remove the singularity in $q$, the problem would be shifted from one in the differential equation to one in the boundary conditions.] Because of this singularity, the problem appears to have a very large perturbation.

For a better uniform solution, it would be desirable if Eq. (C12) were such that the coefficient $2 q$ of $\cos 2 \alpha$ is of a lower order in $\lambda$, rather than being of the same or higher order, as compared with the first two terms. This result could presumably be achieved by choosing better action-angle variables. For example, based on results of Noid et al. who used a curvilinear (parabolic) path, ${ }^{2}$ one might use actions in parabolic coordinates, in which the unperturbed problem is separable and which are appropriate to the observed $^{2}$ shapes of the trajectories. However, the perturbation terms then become more complicated.

The algebraic quantization method involves a matrix diagonalization and so forces the wave function at small $\lambda$ to resemble more closely the unperturbed ones, even for this system. However, the Mathieu functions, which appear as the solution to Eqs. $(\mathrm{Cl2})$ and $(\mathrm{Cl})$ do not resemble the unperturbed ones when $\lambda$ is small, because of the singularity in $q$ at $\lambda=0$ just mentioned. The problem is the same when a uniform semiclassical solution is introduced through a phase integral method. ${ }^{16}$ Results obtained with the latter method and by the Mathieu function method have been shown to be, typically, quite similar. ${ }^{17}$ Use of a suitably chosen path such as that in Ref. 2 can be expected to remove this difficulty. In the spectroscopy literature there are analogous examples of how different approximate results can be obtained merely by using different coordinates. ${ }^{9}$ For example, a second order perturbation treatment gave different results using polar and rectangular coordinates, when certain off-diagonal matrix elements in the Cartesian description were omitted, but the results became identical of course when all such elements were included. ${ }^{9}$

\section{F. Molecular aspects of the Hamiltonian}

The Henon-Heiles Hamiltonian (2.1), which originated in the astronomy literature ${ }^{22}$ in a search of extra constants (hidden constants) of the motion, has since been in widespread use. However, its relation to an actual molecular Hamiltonian does not seem to have been pointed out before and we do so in the present section.

There are many examples of molecules which have two degenerate normal modes. In such cases, the overtones of these modes can be split by perturbations. These perturbations may either contain solely the pair of degenerate normal mode coordinates ${ }^{23}$ or incorporate other normal mode coordinates. ${ }^{24}$ The relevant anharmonicity constants of the molecule can thereby be determined from the positions of the experimental spectral lines.
In order to see the close connection between the present model problem (a Hamiltonian with $C_{3}$ symmetry') and some molecular problems, we examine the Hamiltonian of a molecule with $C_{3 v}$ symmetry and hence with a pair of degenerate normal modes. Denoting the coordinates of those modes by $q_{1}$ and $q_{2}$ one finds that two principal perturbations to its energy levels have the form ${ }^{23}$

$$
V_{1}=\alpha \rho^{3} \cos 3 \varphi+\beta \rho^{4},
$$

where

$$
\rho^{2}=q_{1}^{2}+q_{2}^{2}, \quad \varphi=\arctan \left(q_{2} / q_{1}\right),
$$

and $\alpha, \beta$ are perturbation parameters related to the force constants of the molecule. The first term in $V_{1}$ is the same as the anharmonic potential in the Henon-Heiles Hamiltonian. ${ }^{1}$ This term splits overtones of $A_{1}$ and $E$ symmetry with the same principal quantum number $n$. However, it has been pointed out ${ }^{25}$ that $V_{1}$ does not, in second order, split the $A_{1}$ and $A_{2}$ levels belonging to the same $n$ manifold. That splitting corresponds to the splitting between $l= \pm 3 k$ levels in our treatment, and we have seen earlier that $V_{1}$ causes a splitting in fourth and higher order perturbation theory (Table V). The authors in Ref. 26 added, instead, an additional potential

$$
V_{2}=\gamma \rho^{6} \cos 6 \varphi \text {. }
$$

It has also been shown ${ }^{26}$ that in order to obtain the complete splittings predicted by group theory for the point group $D_{6 h}$ one needs Eq. (6.3) and a $\rho^{12} \cos 12 \varphi$ term in a Hamiltonian. When perturbations of such high order are involved, the highly automated procedures (using algebraic computer language) of BGPT, complemented by uniform approximations when necessary, might provide a viable alternative to straightforward applications of quantum perturbation theory.

The BGPT plus AQ method resembles a quantum mechanical method used by spectroscopists for treating anharmonicities, namely the Van Vleck contact transformation procedure. $^{27.28}$ The latter employs commutators with loworder Hamiltonians to find the necessary generating functions and to successively cause off-diagonal terms to occur only in increasingly higher order.

For systems having degenerate local mode frequencies, such as $\mathrm{H}_{2} \mathrm{O}$, the polar operators' $A Q$ method is not applicable, since the resulting normal modes are not exactly degenerate. (To be sure, the splitting is usually quite small, depending on the mass relations in the molecule.) In the uniform treatment of such systems, ${ }^{29}$ a different perturbation scheme was used instead, utilizing the 1:1 resonance of the local modes.

\section{SUMMARY}

We have seen that in the algebraic quantization method, the choice of coordinate system facilitates the calculation. The choice is more a matter of computational convenience. The results appear to be better that perturbative semiclassical procedures to the same order; the algebraic quantization accounts better for the quantum mechanics than do semiclassical methods. Of course, semiclassical quantization is readily adapted to the shapes of the trajector- 
ies, either as in Refs. 1 and 2 or, in conjunction with perturbation theory, as in Ref. 10 (cf. present Sec. V D).

In semiclassical quantization, the results can depend on the coordinate system or quantization path chosen. A bad choice, which ignores the shape of the trajectories, by using, e.g., Cartesian actions or any constant linear combination of Cartesian actions for precessing trajectories in the HenonHeiles system, appears to give poorer results. In the case of the US method, perhaps if one went to higher order (i.e., higher cosine terms) and if the series did not diverge this sensitivity would be less. The adverse consequences of using inappropriate coordinates can be removed when one uses phase integral paths that take into account the good quantum numbers of the system.

One can also expect that when the curved shape of the trajectories is not too distorted from the shape of a rectangle, Cartesian actions may be quite useful. An example might be some 2:1 Fermi resonance system whose trajectories undergo only a relatively weak libration of their figure-eightlike motion instead of the precessing type motion of the ellipse-like trajectories of 1:1 resonance systems.

These remarks concerning the AQ and US methods, Cartesian actions, and calculations based on the shape of the trajectories or (cf. Figs. 8 and 13 of Ref. 2) wave functions are expected to apply also to other methods for approximate quantization, such as the adiabatic separation method. ${ }^{30}$

\section{ACKNOWLEDGMENT}

We are pleased to acknowledge the support of this research by the National Science Foundation.

\section{APPENDIX A. AQ CALCULATED SPLITTINGS OF $l= \pm 3 k$ LEVELS USING POLAR OPERATORS' BASIS}

We first use the off-diagonal part $N_{5}$ of $H_{4}$, given by Eq. (3.10), to calculate the splittings of the $l= \pm 3 k$ states. One first notes that

$N_{1}^{3}|n, l\rangle=-\frac{1}{8} A_{1}|n, l+6\rangle-\frac{1}{8} A_{2}|n, l-6\rangle-\frac{3}{8} A_{3}|n, l\rangle$,

where

$A_{1}=\left\{(n-l)(n+l+6)\left[n^{2}-(l+4)^{2}\right]\left[n^{2}-(l+2)^{2}\right]\right\}^{1 / 2}$,

$A_{2}=\left\{(n+l)(n-l+6)\left[n^{2}-(l-4)^{2}\right]\left[n^{2}-(l-2)^{2}\right]\right\}^{1 / 2}$,

$A_{3}=\sqrt{(n-l)(n+l+2)}\left[n^{2}-l^{2}+2(n-l)\right]$

$+\sqrt{(n+l)(n-l+2)}\left[n^{2}-l^{2}+2(n+l)\right]$.

$N_{0}$ and $N_{1}$ in Eq. (3.10) commute, but $N_{2}^{2}$ and $N_{1}$ do not. One then takes the Weyl transform of $N_{2}^{2} N_{1}$ in the version

$$
N_{2}^{2} N_{1} \leftrightarrow\left(N_{2}^{2} N_{1}+2 N_{2} N_{1} N_{2}+N_{1} N_{2}^{2}\right) / 4 .
$$

Using Eqs. (3.8), (A1), and (A2) the effect of the $N_{5}$ in Eq. (3.10) on $|n, l\rangle$ is then found to be

$$
N_{5}|n, l\rangle=-\frac{1}{2} A_{1}|n, l+6\rangle-\frac{1}{2} A_{1}|n, l-6\rangle,
$$

where the first term on the right-hand side is replaced by zero when $n<|l+6|$, and the second term is replaced by zero when $n<|l-6|$.

All operators except $N_{5}$ in $H_{4}$ are diagonal, so that in contrast to the situation for Cartesian operators one can identify an operator that causes the splitting, and can proceed directly to evalute it by degenerate perturbation theory. For instance, in the case of $n=3$, only a $2 \times 2$ diagonalization of the off-diagonal operator [cf. Eqs. (2.10) and (3.10)]

$$
H_{4}^{\mathrm{OD}} \equiv 7 N_{\mathrm{s}} / 18
$$

in the subspace $|n, l\rangle=|3, \pm 3\rangle$ is required. Using the AQ method the splitting is then given as 0.0029 , as in Table II. The same calculation requires a lengthier $4 \times 4$ diagonalization in the Cartesian basis set. If one takes into account only the $N_{5}$ that occurs in $H_{6}$ in Eq. (2.11), the off-diagonal element in $H_{6}$ becomes

$$
H_{6}^{\mathrm{OD}} \equiv 2093 N_{5} N_{0} / 2160 \text {. }
$$

[The remaining two off-diagonal terms in Eq. (2.11) are multiplied by small coefficients and are omitted here for simplicity.] With this addition to Eq. (A4) the splitting of the $|3, \pm 3\rangle$ state is the 0.0033 in Table II.

The operator (A5) can also be used to obtain an estimate for the $l= \pm 6$ splitting by diagonalizing it using the $l=0$ and $l= \pm 6$ states. The result of this $3 \times 3$ diagonalization for $n=6$ is the 0.0005 in Table II. This is only an estimate because $H_{6}$ contains other nondiagonal operators that couple $l= \pm 3$ or $l= \pm 6$ states indirectly (in $\Delta l$ units of 2 or 4 ), and a more elaborate diagonalization would include them. However, the results using Eqs. (A4) and (A5) in a $2 \times 2$ diagonalization for $l= \pm 3$ states are quite acceptable even for high $n$ [error for $n=3$ to 9 is about $10 \%$, better than the corresponding uniform semiclassical result ${ }^{11}$ that also was to the same order than the result based on Eqs. (4.18) and (4.19)]. The error in splitting of the $(6, \pm 6)$ states in Table $V$ using the $3 \times 3$ diagonalization $(\sim 17 \%)$ was also less by a factor of 2 than that obtained in the US result in Ref. 11 [cf. the present Tables II and $V$ for the $(6, \pm 6)$ pair].

\section{APPENDIX B. UNIFORM SEMICLASSICAL QUANTIZATION USING CARTESIAN ACTIONS}

When the transformation

$q_{i}=\left(2 I_{i}\right)^{1 / 2} \sin \varphi_{i}, \quad p_{i}=\left(2 I_{i}\right)^{1 / 2} \cos \varphi_{i}, \quad i=1,2$,

where $I_{i}, \varphi_{i}$ are Cartesian actions and their conjugate angles, is substituted into the Hamiltonian formed by $H_{0}$ and $\mathrm{H}_{2}$ in Eqs. (2.6) and (2.9), one obtains

$$
\begin{aligned}
H\left(I_{i}, \varphi_{i}\right)= & I_{1}+I_{2}-\lambda^{2}\left[5\left(I_{1}^{2}+I_{2}^{2}\right) / 2-2 I_{1} I_{2}\right. \\
& \left.+7 I_{1} I_{2} \cos 2\left(\varphi_{1}-\varphi_{2}\right)\right] .
\end{aligned}
$$

With the canonical transformation (in analogy with Ref. 10)

$$
\begin{array}{ll}
\varphi_{1}=(\theta-\alpha) / 2, & I_{1}=I-I_{\alpha}, \\
\varphi_{2}=(\theta+\alpha) / 2, & I_{2}=I+I_{\alpha},
\end{array}
$$

one obtains

$$
H=2 I-\frac{\lambda^{2}}{6}\left[3 I^{2}+7 I_{\alpha}^{2}+7 I_{1} I_{2} \cos 2 \alpha\right] .
$$

In the coefficient of the $\cos 2 \alpha$ term, average actions $I_{1}, I_{2}$ will be used, obtained as arithmetic averages of the actions $I_{i}$ between the states that are coupled by the $\cos 2 \alpha$ term. This procedure has been discussed elsewhere. ${ }^{12}$ Rearranging Eq. (B4), 


$$
I_{\alpha}^{2}+\bar{I}_{1} \bar{I}_{2} \cos 2 \alpha+\lambda^{2} I^{2} / 2+{ }_{7}^{6}(E-2 I) / \lambda^{2}=0,(\mathrm{~B} 5)
$$

and making the replacement

$$
I_{\alpha} \rightarrow-i d / d \alpha,
$$

one obtains a differential equation for the wave function $\psi(\alpha)$ :

$$
\begin{aligned}
& d^{2} \psi(\alpha) / d \alpha^{2} \\
& +\left[\frac{6}{\gamma}\left(2 I-\lambda^{2} I^{2} / 2-E\right) / \lambda^{2}-\bar{I}_{1} \bar{I}_{2} \cos 2 \alpha\right] \psi(\alpha)=0 .
\end{aligned}
$$

The parameters of the Mathieu equation (4.13) are thus

$$
q=\bar{I}_{1} \bar{I}_{2} / 2, \quad a=6\left(2 I-\lambda^{2} I^{2} / 2-E\right) / \lambda^{2},
$$

giving the energy as

$$
E=2 I-\lambda^{2}\left(I^{2} / 2+7 \alpha_{v} / 6\right),
$$

using Eq. (B8). The boundary conditions on Eq. (B7) are obtained from those in the product of wave functions of $\varphi_{1}$ and $\varphi_{2}$. Using Eq. (B3) one then finds

$$
\psi(\alpha+\pi)=\exp \left[i \pi\left(n_{2}-n_{1}\right) / 2\right] \psi(\alpha),
$$

where $n_{1}, n_{2}$ are the Cartesian quantum numbers in the state considered. By Floquet's theorem ${ }^{19}$ [Eqs. (4.15) and (4.16)], the order of relevant solutions is therefore

$$
v=\left(n_{2}-n_{1}\right) / 2 \text {. }
$$

The energy levels calculated using Eq. (B9) are not as accurate as the ones calculated to order $\mathrm{H}_{2}$ using the uniform semiclassical quantization and the $|n, l\rangle$ basis (Sec. IV). The reasons for this discrepancy are to be sought in the nature of the approximations made to put the perturbation Hamiltonian (B2) in the form of a Mathieu equation (cf. the text for the "order" of goodness of $N_{1}$ and $N_{2}$ ).

One expects that Eq. (B9) will be most useful when the effects of the perturbation in Eq. (4.13) are such that the $a$ is dominated by the $v^{2}$ (in $a_{v}$ ) rather than by the barrier $q$. In Ref. 12, for example, the $q$ was proportional to $\lambda$, and hence was relatively small. However, the $q$ in the present Cartesian action problem is of the same order in $\lambda$ in Eq. (B5) as the $I_{\alpha}^{2}$ term, due to the choice of the zero order variables, and so Eq. (B5) no longer resembles a perturbation equation for $I_{\alpha}$. A more drastic example of such behavior is found in the Fermi resonance problem discussed at the end of Sec. IV and in Appendix C.

The results of solving Eqs. (B7) and (B10) were given in Table VI. It is useful to compare these results with those obtained by matrix diagonalization of Eq. (B4), since matrix diagonalization was the method used in Refs. 3 and 4 . We consider the three states that arise from the three unperturbed states $\left|n_{1}, n_{2}\right\rangle=|2,0\rangle,|1,1\rangle$, and $|0,2\rangle$. They all have $n=2$ and $I=3 / 2$. Transcribing Eq. (B4) one obtains

$$
H=2 I-\frac{1}{6} \lambda^{2}\left(3 I+7 I_{\alpha}^{2}\right)-\frac{7}{3} \lambda^{2} q \cos 2 \alpha .
$$

The last term couples the $|2,0\rangle$ and $|0,2\rangle$ states, and the offdiagonal matrix element of $\cos 2 \alpha$ is (using the unperturbed semiclassical wave functions) given by

$$
\langle 2,0|\cos 2 \alpha| 0,2\rangle=\frac{1}{2 \pi} \int_{0}^{2 \pi} e^{2 i \alpha} \cos 2 \alpha d \alpha=1 / 2 .
$$

The approximate energy for the perturbed $|1,1\rangle$ state $E_{(1,1)}$ is $2 I-\lambda^{2} I^{2} / 2$ i.e., 2.9859 when $I=3 / 2$ and $\lambda^{2}=0.0125$. Similarly, $E_{(2,0)}=E_{(0,2)}=2.9714$. The diagonalization of the $H$ appearing in Eq. (B2), in the subspace of the three $n=2$ states, leads to the results labeled $D$ in Table VI.

\section{APPENDIX C. UNIFORM SEMICLASSICAL QUANTIZATION OF THE FERMI RESONANCE USING CARTESIAN ACTIONS}

An extreme case of how the choice of good action variables affects the simple uniform scheme outlined in Sec. II is given by the Hamiltonian

$H=\left(P_{1}^{2}+P_{2}^{2}+\omega_{1}^{2} Q_{1}^{2}+\omega_{2}^{2} Q_{2}^{2}\right) / 2+\lambda Q_{1}\left(Q_{2}^{2}+\eta Q_{2}^{2}\right)$

with $\omega_{1}=2 \omega_{2}$. (That is, it shows a 2:1 or Fermi resonance. ${ }^{2.31}$ ) The Birkhoff-Gustavson scheme and the notation is the same as in Appendix A of Ref. 12. The Hamiltonian is, with $\omega_{1}=\omega$,

$H=\frac{1}{2}\left(P_{1}^{2}+\omega^{2} Q_{1}^{2}+P_{2}^{2}+\frac{\omega^{2} Q_{2}^{2}}{4}\right)+\lambda Q_{1} Q_{2}^{2}+\eta \lambda Q_{1}^{3}$,

and is transformed into the Hamiltonian (C3) by the transformation $P_{i}=\omega_{i}^{1 / 2} p_{i}, Q_{i}=q_{i} / \omega_{i}^{1 / 2}$.

$$
H=H^{(2)}+H^{(3)} \text {, }
$$

where

$$
\begin{aligned}
& H^{(2)}=\frac{\omega}{2}\left[\left(p_{1}^{2}+q_{1}^{2}\right)+\frac{1}{2}\left(p_{2}^{2}+q_{2}^{2}\right)\right], \\
& H^{(3)}=\left(2 \lambda q_{1} q_{2}^{2}+\eta \lambda q_{1}^{3}\right) \omega^{-3 / 2} .
\end{aligned}
$$

The Birkhoff-Gustavson scheme provides an algorithm to obtain the generating functions $W^{(i)}$. With their help the new Hamiltonian becomes

$$
\Gamma=\sum_{i=2}^{\infty} \Gamma^{(i)} \text {. }
$$

By the processes outlined in Ref. 12, one obtains

$$
\Gamma^{|3|}=\lambda\left[2 p_{1} p_{2} q_{2}-q_{1}\left(p_{2}^{2}-q_{2}^{2}\right)\right] \omega^{-3 / 2} / 2
$$

and

$$
\begin{aligned}
W^{(3)}= & \lambda\left[p_{1}\left(3 p_{2}^{2}+5 q_{2}^{2}\right)+2 q_{1} p_{2} q_{2}\right. \\
& \left.+4 \eta\left(2 p_{1}^{3}+3 p_{1} q_{1}^{2}\right) / 3\right] \omega^{-5 / 2} / 4 .
\end{aligned}
$$

From this generating function $W^{(3)}$ one obtains

$$
\begin{aligned}
\Gamma^{(4)}= & \lambda^{2}\left[15 \eta^{2}\left(p_{1}^{2}+q_{1}^{2}\right)^{2}+9\left(p_{2}^{2}+q_{2}^{2}\right)^{2}\right. \\
& \left.+4(6 \eta+1)\left(p_{1}^{2}+q_{1}^{2}\right)\left(p_{2}^{2}+q_{2}^{2}\right)\right] / 16 \omega^{4},
\end{aligned}
$$

thereby arriving at the same Hamiltonian as that found by Sanders ${ }^{3}$ by averaging. The procedure for going from Eqs. (C5), (C6), and (C8) to the corresponding Mathieu equation has been treated in Ref. 12. Using the canonical transformation

$$
\begin{aligned}
& 2 \alpha=\varphi_{1}-2 \varphi_{2}+\pi / 2, \quad I_{\alpha}=2 I_{1}, \\
& \theta=\varphi_{2}, \quad I=2 I_{1}+I_{2},
\end{aligned}
$$

one obtains, from Eq. (C5) through fourth order, with Eqs. (C6) and (C8),

$$
\begin{aligned}
H= & \omega I / 2+\lambda\left(2 I_{1} I_{2}^{2} / \omega^{3}\right)^{1 / 2} \cos 2 \alpha \\
& +\lambda^{2} \omega^{-4}\left[-15 \eta^{2} I_{\alpha}^{2} / 16-(6 \eta+1) I_{\alpha}\left(I-I_{\alpha}\right) / 2\right. \\
& \left.-9\left(I-I_{\alpha}\right)^{2} / 4\right] .
\end{aligned}
$$


Rearranging one obtains

$$
\begin{aligned}
\lambda^{2} \omega^{-4} & \left(15 \eta^{2}-48 \eta+28\right) I_{\alpha}^{2} / 16+\lambda^{2} \omega^{-4}(3 \eta-4) I_{\alpha} I \\
& +9 I^{2} \lambda^{2} \omega^{-4} / 4+E-\omega I / 2 \\
& -\lambda\left(2 I_{1} I_{2}^{2} / \omega^{3}\right)^{1 / 2} \cos 2 \alpha=0 .
\end{aligned}
$$

The latter can be rewritten as

$$
I_{\alpha}^{2}-2 \beta I_{\alpha}+2 q \cos 2 \alpha+D E-C=0
$$

for $\lambda \neq 0$, with

$$
\begin{aligned}
\beta & \equiv \frac{I}{2}(4-3 \eta) / G, \quad q \equiv-\lambda^{-1}\left(\bar{I}_{1} \bar{I}_{2}^{2} \omega^{5} / 2\right)^{1 / 2} / G, \\
C & \equiv\left[I \omega^{5} / 2 \lambda^{2}-9 I^{2} / 4\right] / G, \quad D \equiv\left(\lambda / \omega^{2}\right)^{-2} / G, \\
G & \equiv 15 \eta^{2} / 16-3 \eta+7 / 4 .
\end{aligned}
$$

We have used average values for the $I_{i}$ in Eq. $(\mathrm{C} 13)$ for $q$ to make $q$ a constant. Equation $(\mathrm{C} 12)$ can then be converted to a Mathieu equation

$$
\frac{d^{2} F}{d \alpha^{2}}+\left(a_{v}-2 q \cos 2 \alpha\right) F=0
$$

for the function $F$. $F$ is related to the wave function $\psi(\alpha)$ by

$$
F(\alpha)=\exp [i(1-\beta)] \psi(\alpha) .
$$

The $a_{v}$ in Eq. $(\mathrm{C} 16)$ is related to the energy $E$ via

$$
a_{v}=C-D E+\beta^{2} \text {. }
$$

The order $v$ of the solutions is given by

$$
v=2 n_{1}+1-\beta,
$$

following the argument used to treat an avoided crossing Hamiltonian in Ref. 12. With this $v$ the corresponding characteristic values are found and the quantized energies determined from them using Eq. (C18).

In contrast to that equation or the other differential equations appearing in this work, Eq. (C12) has a singularity at $\lambda=0$. For $\lambda \neq 0$ and small, some of the coefficients become very large. The results are discussed in the text. Using Eqs. (C13)-(C19), one can still calculate energy levels following the procedure outlined in Ref. 12. In those calculations, one typically has to contend with rather large $q$ (of order of $10)$ and can use the asymptotic form for the eigenvalues ${ }^{13}$

$$
\begin{aligned}
\alpha_{v}(q) \sim & -2 q+2(2 v+1) q^{1 / 2} \\
- & {\left[(2 v+1)^{2}+1\right] / 8+O\left(q^{-1 / 2}\right) . }
\end{aligned}
$$

To obtain the results in Table VII the parameters in Eqs. $(\mathrm{C} 12)-(\mathrm{C} 15)$ had the values of $q=62.8200, \beta=5.3509$, $D=1471.6519, C=4607.7766$, and $G=1.6315$ appropriate for the relevant values of the original parameters in footnote a of Table VII.

'D. W. Noid and R. A. Marcus, J. Chem. Phys. 67, 559(1977); D. W. Noid, M. L. Koszykowski, M. Tabor, and R. A. Marcus, ibid. 73, 6169 (1980). ${ }^{2}$ D. W. Noid, M. L. Koszykowski, and R. A. Marcus, J. Chem. Phys. 71, 2864 (1979). A sign misprint occurs in Eq. (1): $-\alpha$ should be $+\alpha$. All results in this reference were for this (sign corrected) equation. See D. $M$. Wardlaw, D. W. Noid, and R. A. Marcus, J. Phys. Chem. 88, 536 (1984), Ref. 37.

${ }^{3} J$. A. Sanders, J. Chem. Phys. 74, 5733 (1981), who used the "averaging" perturbation method (Ref. 6 below).

${ }^{4} M$. Robnik, J. Phys. A 17, 109 (1984), who used the Birkhoff-Gustavson perturbation method (Ref. 5 below).

${ }^{5}$ F. G. Gustavson, Astron. J. 71, 670 (1966).

${ }^{6}$ N. N. Bogoliubov and Y. A. Mitropolsky, Asymptotic Methods in Nonlinear Oscillations (Hindustan, Delhi, 1961).
'S. R. de Groot and L. G. Suttorp, Foundations of Electrodynamics (Elsevier, New York, 1972)

${ }^{8}$ J. D. Louck and W. H. Shaffer, J. Mol. Spectrosc. 4, 285 (1960).

${ }^{9}$ E.g., J. Overend, Spectrochim. Acta Part A 32, 1581 (1976).

${ }^{10}$ R. T. Swimm and J. B. Delos, J. Chem. Phys. 71, 1706 (1979).

${ }^{11} \mathrm{C}$. Jaffe and W. P. Reinhardt, J. Chem. Phys. 77, 5191 (1982); 71, 1862 (1979).

${ }^{12}$ T. Uzer, D. W. Noid, and R. A. Marcus, J. Chem. Phys. 79, 4412 (1983).

${ }^{13} \mathrm{~N}$. W. McLachlan, Theory and Applications of Mathieu Functions (Clarendon, Oxford, 1947).

${ }^{14} \mathrm{G}$. Herzberg, Molecular Spectra and Molecular Structure. II. Infrared and Raman Spectra of Polyatomic Molecules (Van Nostrand, Princeton, 1945), p. 210. For example, in Table IV of the present paper the fundamental transition $(0,0) \rightarrow(1 \pm 1)$ is not split.

${ }^{15}$ K. S. J. Nordholm and S. A. Rice, J. Chem. Phys. 61, 203 (1974).

${ }^{16}$ W. H. Miller, J. Chem. Phys. 48, 1651 (1968).

${ }^{17}$ J. N. L. Connor, T. Uzer, R. A. Marcus, and A. D. Smith, J. Chem. Phys. 80, 5095 (1984).

${ }^{18}$ One uses an argument similar to that used in Sec. IV of Ref. 12. Using Eq. (4.12) one sees that $\psi(\alpha+\pi)$ equals $F\left(\varphi+\frac{1}{3} \pi\right)$. Apart from a preexponential factor, the semiclassical wave function is $F(\varphi) \sim \exp i\left[\int_{0}^{\varphi} I_{\varphi} d \varphi\right]$. However, one sees from Eq. (4.8) that $I_{\varphi}(\varphi+\pi / 3)=I_{\varphi}(\varphi)$ and hence that $\int I_{\varphi} d \varphi$ is the same regardless of whether the limits are $\left(\varphi, \varphi+\frac{1}{3} \pi\right)$ or $\left(0, \frac{1}{3} \pi\right)$. Thereby, one finds that

$$
\begin{gathered}
F\left(\varphi+\frac{1}{3} \pi\right) \sim \exp i\left[\int_{0}^{\varphi} I_{\varphi} d \varphi\right] \exp i \int_{0}^{\pi / 3} I_{\varphi} d \varphi \\
=F(\varphi) \exp \frac{i}{3} \int_{0}^{\pi} I_{\varphi} d \varphi .
\end{gathered}
$$

In a quantum state, $\int_{0}^{\pi} I_{\varphi} d \varphi=l \pi$, and thus $F\left(\varphi+\frac{1}{3} \pi\right)=e^{i l \pi / 3} F(\varphi)$. Equations (4.16) and (4.17) then follow.

${ }^{19} \mathrm{G}$. Blanch, in Handbook of Mathematical Functions, edited by $\mathrm{M}$. Abramowitz and I. A. Stegun (Dover, New York, 1969), p. 727.

${ }^{20}$ Expansions of $a_{v}$ in terms of $q$ and $v$ exist for small $q$ (Refs. 13 and 19). For moderate or large $q$ tables can be consulted which make use of continued fraction methods. [See T. Tamir, Math. Comput. 16, 100 (1962) for $q \leqslant 2.5$.] When $v$ is an integer $r$, it is convenient to associate $v=r$ with the characteristic value $a_{r}(q)$ and $v=-r$ with $b_{r}(q)$, for which similar types of expansions and tables exist (as in Refs. 13,19 and in T. Tamir, loc. cit.). For $q>10$, the asymptotic form given by Eq. (C20) can be used.

${ }^{21}$ This discrepancy can be verified using

$$
\begin{aligned}
& N_{2}\left|n_{1}, n_{2}\right\rangle=i\left[a\left|n_{1}-1, n_{2}+1\right\rangle-b\left|n_{1}+1, n_{2}-1\right\rangle\right] \text {, } \\
& N_{3}\left|n_{1}, n_{2}\right\rangle=a\left|n_{1}-1, n_{2}+1\right\rangle+b\left|n_{1}+1, n_{2}-1\right\rangle \text {, } \\
& \text { where } a=\sqrt{n_{1}\left(n_{2}+1\right)} \text { and } b=\sqrt{n_{2}\left(n_{1}+1\right)} \text {. It follows that } \\
& N_{3}^{2}\left|n_{1}, n_{2}\right\rangle=\left(n_{1}+n_{2}+2 n_{1} n_{2}\right)\left|n_{1}, n_{2}\right\rangle \\
& +\left[n_{2}\left(n_{2}-1\right)\left(n_{1}+1\right)\left(n_{1}+2\right)\right]^{1 / 2}\left|n_{1}+2, n_{2}-2\right\rangle \\
& +\left[n_{1}\left(n_{1}-1\right)\left(n_{2}+1\right) \mid\left(n_{2}+2\right)\right]^{1 / 2}\left|n_{1}-2, n_{2}+2\right\rangle,
\end{aligned}
$$

and that $N_{2}^{2}\left|n_{1} n_{2}\right\rangle$ is given by a similar expression, but with the coefficients of $\left|n_{1}+2, n_{2}-2\right\rangle$ and $\left|n_{1}-2, n_{2}+2\right\rangle$ having a negative sign. A fuller discussion of the discrepancy caused by Eq. (2.8) is given in J. R. Shewell, Am. J. Phys. 27, 16 (1959).

${ }^{22} \mathrm{M}$. Henon and C. Heiles, Astron. J. 69, 73 (1964).

${ }^{23}$ See, for example, T. D. Kolomiitsova and D. N. Shchepkin, Opt. Spektrosk. 32, 915 (1972) [Opt. Spectrosc. 32, 488 (1972)].

${ }^{24}$ See, for example, L. V. Belyavskaya, V. S. Kravchenko, N. K. Morozova, and V. P. Morozov, Opt. Spektrosk. 38, 1105 (1975) [Opt. Spectrosc. 38, $638(1975)]$

${ }^{25}$ J. J. Lopez-Gonzalez and J. F. Arenas, An. Quim. 77, 51 (1981).

${ }^{26}$ J. J. Lopez-Gonzalez and J. F. Arenas, An. Quim. 77, 21 (1981).

${ }^{27}$ H. H. Nielsen, in Handbuch der Physik, edited by S. Flugge (Springer, Berlin, 1959), Vol. 39; D. Papousek and M. R. Aliev, Molecular Vibrational-Rotational Spectra. Studies in Physical and Theoretical Chemistry (Elsevier, New York, 1982), Vol. 17.

${ }^{28}$ S. Califano, Vibrational States (Wiley, New York, 1976).

${ }^{29}$ E. L. Sibert, W. P. Reinhardt, and J. T. Hynes, J. Chem. Phys. 77, 3583 (1982); 77, 3595 (1982).

${ }^{30}$ R. S. Caswell and M. Danos, J. Math. Phys. 11, 349 (1970); G. Hose, H. S. Taylor, and Y. Y. Bai, J. Chem. Phys. 80, 4363 (1984), and references cited therein.

${ }^{31}$ J. D. Louck, M. Moshinsky, and K. Wolf, J. Math. Phys. 14, 692 (1973); 14, 696 (1973); C. Boyer and K. B. Wolf, ibid. 16, 2215 (1975). 